\title{
Postures and Motions Library Development for Verification of Ground Crew Human Factors Requirements
}

\author{
Damon Stambolian and Gena Henderson, Ph.D. \\ NASA Kennedy Space Center \\ Mariea Dunn Jackson, Ph.D. and Charles Dischinger \\ NASA Marshall Space Flight Center
}

\begin{abstract}
Spacecraft and launch vehicle ground processing activities require a variety of unique human activities. These activities are being documented in a primitive motion capture library. The library will be used by human factors engineering analysts to infuse real to life human activities into the CAD models to verify ground systems human factors requirements. As the primitive models are being developed for the library, the project has selected several current human factors issues to be addressed for the Space Launch System (SLS) and Orion launch systems. This paper explains how the motion capture of unique ground systems activities is being used to verify the human factors engineering requirements for ground systems used to process the SLS and Orion vehicles, and how the primitive models will be applied to future spacecraft and launch vehicle processing.
\end{abstract}

\section{INTRODUCTION}

There are over 40 subsystems being developed for the future SLS and Orion Launch Systems at Kennedy Space Center [1].

An independent NASA Engineering and Safety Center (NESC) risk mitigation project was initiated to create a library of postures (body positions) and motions of CAD-based human models for use in evaluating worksites for ground processing of flight hardware. The scope of this assessment was to develop a library of human model motion and posture behavior primitives, which are the basic routines or segments used to construct more complex models of postures and motions in the design and analysis of ground processing and maintenance tasks. The primary utility of these primitives will be to launch system design by NASA and its contractors and commercial partners.

CAD tools are a critical part of design and early down selection. Current human models lack inherent awareness of how humans actually perform motions required for simple tasks.

Programmatic requirements documents and NASA standards provide requirements for design of systems for ground processing and maintenance. They call for design decisions that enable work that is efficient and that protects flight hardware by appropriate sizing and location of work stations for hatches, Line Replaceable Units, connectors, fuel loading, and so forth.

The Human Engineering Modeling and Performance (HEMAP) motion capture laboratory at Kennedy Space Center (KSC) worked with the human factors design team in the Virtual Environments Lab (VEL) at Marshall Space Flight Center (MSFC) to develop a library of common ground processing actions, so that tasks can be quickly modeled based on validated primitives. Human flight programs assume that these models are available (which they are not) for design and verification. A great deal of time and resource costs can be saved through digital design and through designing for processing.
This project's efforts are to create a library of such postures and motions that can be called upon by analysts for any design effort. The library is useful to designers who must decide whether to design a worksite for 1,2 , or more personnel and whether special ground support equipment is required; these and similar decisions directly affect protection of flight hardware and thus, flight safety. The project will establish the reliability (predictive power) of the models in the library as well as constraints. The library is planned to be made available for application by NASA and its contractors and commercial partners. The project aims to increase ground personnel safety at work sites and to reduce the risk of damage to flight hardware by providing human models that accurately represent the way that humans perform ground processing and maintenance tasks. This gives an improved level of reliability to the analyses that evaluate the design for task performance, plus eliminates significant key framing resources that would otherwise be required with traditional human digital modeling.

\section{Human Engineering Modeling And Performance (HEMAP) AND VIRTUAL ENVIRONMENTS LAB (VEL)}

The HEMAP Lab at KSC has deployed motion capture technology of single or multiple persons and objects $[2,3]$. The lab, originally funded as a safety enhancement, has also benefited from Research and Development (R\&D) activities which now afford live data streaming, real-time ergonomic evaluations, integrated biomedical monitoring, and collaboration of raw motion data with other interacting motion capture labs. Customer provided computer environments and basic physical interactive or stationary work envelope mockups also can be integrated to understand and assess situational awareness aspects, including human behavior tendencies that cannot also be modeled with created digital avatars or without motion tracking. HEMAP's focus on both spacecraft assembly and ground processing operations has provided evaluations and recommendation considerations affecting Space Shuttle as well as Orion, or Multi-Purpose Crew Vehicle (MPCV) tasks 
including design and training aspects. HEMAP utilizes specialized products that were chosen based on functionality, including software and fixed based hardware (e.g., visible red cameras), along with hand-tracking gloves to assess fine motor dexterity of the hands.

The VEL at MSFC provides capabilities similar to HEMAP's, but with a slightly different configuration. The VEL uses Siemens Jack products (shortened to JACK) human modeling software and the Motion Analysis motion capture system to conduct analyses. The library will contain data captured by integrating the Cortex files from the motion capture software into the JACK virtual environment. The VEL has successfully demonstrated the capability to collaborate real-time with HEMAP in data capture and exchange. This is significant for distributed, collaborative analysis of multi-person tasks. During the Constellation Program, the VEL conducted human modeling analyses of the assembly and integration of the Ares launch vehicle, which contributed greatly to the appropriate implementation of human factors requirements in the design. More recently, the VEL has conducted trade studies and requirements development for SLS and the element design trade studies and for Deep Space Habitat and Deep Space Module design studies.

JACK provides the following capabilities: Scalable human figures that are anthropometrically and biomechanically accurate; Support ergonomic analysis of your global workforce using country-specific population databases; Comprehensive suite of ergonomic analysis tools; Advanced posture algorithm that can also analyze how a body reacts to exerted force in a specific direction; Manage a wide range of workplace scenarios that include working at different levels of elevation, stairs and ramps Eye-view windows and field-of-vision analysis; Reach envelopes for fast workplace configuration; Movie and screen captures for documentation and presentations; and Broad virtual reality motion capture hardware support. Live or postprocessing ergonomic analysis may include Lower Back Analysis (LBA), Rapid Upper Limb Assessment (RULA), and Static Strength Prediction (SSP), Fatigue Analysis, and other key tools.

\section{APPROACH}

Postures and motions collected were based on inputs from requirements, design, modeling and simulation, as well as human factors experts. Candidate tasks were identified and associated postures and motions were captured in the VEL using planned experimentation as well as male and female participants of varying anthropometric criteria. During task execution, ergonomic results, images, screenshots, videos, and raw motion data were collected and then combined with user and human factors inputs to populate the library. Ultimately, it is expected that dozens of captures will be performed using a variety of anthropometric, gender, and skilled participants, demonstrating varieties of postures and motions.

Subjects for this work were recruited from the current technician workforce and engineers at MSFC and at KSC. Engineers have the design understanding of these new systems, and the technicians have the tacit knowledge of pervious processing problems. Combining the two groups as subjects will promote collaborative engineering solutions. Some participants may not have the hands on experience, and this will be recorded.

\section{Problem Description, Proposed Solutions, AND Risk ASSESSMENT}

Modeling and simulation (M\&S) and visualization tools are a critical part of the system design process and early down selection between design options during trade studies. The same tools are ultimately used for requirements verification. In the area of ground processing and maintenance, M\&S supports design decisions that enable work that is safe, efficient, and affordable by adequate sizing and location of hatches, work areas, line replaceable units, connectors, etc. Ground processing work task analyses are often performed using human models that are posed by the analyst based on his or her judgment of the correct body position and motion required to perform the task. A more reliable analysis would incorporate data on body positions and motions gathered from knowledgeable ground personnel. Motion capture technology provides methods for doing this.

This effort supports NESC in the development of human model "primitives" of motions and postures utilized by ground crews during specific tasks during launch vehicle and spacecraft processing. Making the library available to analysts for design, development, and verification of worksites for launch system ground processing and maintenance supports verification of applicable human-system integration requirements for SLS, MPCV, and Ground Systems Development and Operations (GSDO).

For this project, the library will not include microgravity worksites. Once the library has been developed and is proven for ground, future projects will then expand into the microgravity worksites. In this environment, the neutral body postures and translation modes are vastly different and need to be available for designers of non 1-G environments [1].

\section{APPLIED METHODOLOGY}

The project steering team, consisting of various NASA center civil servants and contractor lab/team specializing in motion capture and human factors assessments, established the following areas for completing the project.

- Identify the human-related tasks with significance to multiple stakeholders, including programs and requirements as well as designers or operations end-users that would benefit from found results and data.

- Identify human modeling components having applicability to aerospace vehicles and associated ground processing to determine key operational capabilities of single or multiple persons, including use of motion tracking peripherals and mockups, as applicable. 
- Evaluate actual tasks and software-supported ergonomics and human factors simulations, relying on human factors subject matter experts to make recommendations for the library.

- Develop a posture/motion library to contain results of motion capture studies including human factors results and raw motion data.

- Document the results in a final report.

\section{TASK AND POSTURE/MOTION SELECTION}

Prior to designing human tasks to perform and evaluate, reviews of human-related tasks were conducted based on lessons learned from Shuttle, Orion, and other previous hardware and ground processing activities [4]. Requirements for forthcoming spacecraft vehicles and ground operations designs and tasks also were evaluated.

At a kickoff workshop, briefings on requirements for ground processing issues and difficulties, design requirements for ground processing worksites, and motion capture technologies in general and the contractor capabilities in particular, were communicated. The team then developed and prioritized a list of tasks to be analyzed based on the following criteria:

- Importance to stakeholders and programs

- Concerns to M\&S Designers

- Applicability to programs, vehicles, facilities, GSE

- Feasibility

- Frequency/Complexity/Hazard Levels

\section{ShUTtLe Processing Lessons LeARNed}

Ground processing tasks associated with Shuttle involved a myriad of various postures, performed by single or multiple person task teams. Modifications to GSE or infrastructure improvements over decades improved human factors and reduced risks. Due to facility structure, hardware configuration, and other various, many others could not be improved to eliminate all human factors or safety risks. The following Figures 1 to 5 show some of the Space Shuttle postures.
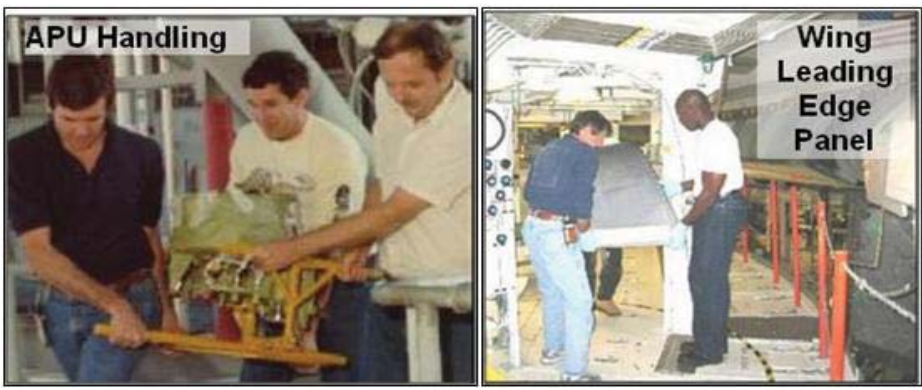

Figure 1 Carrying/Handling
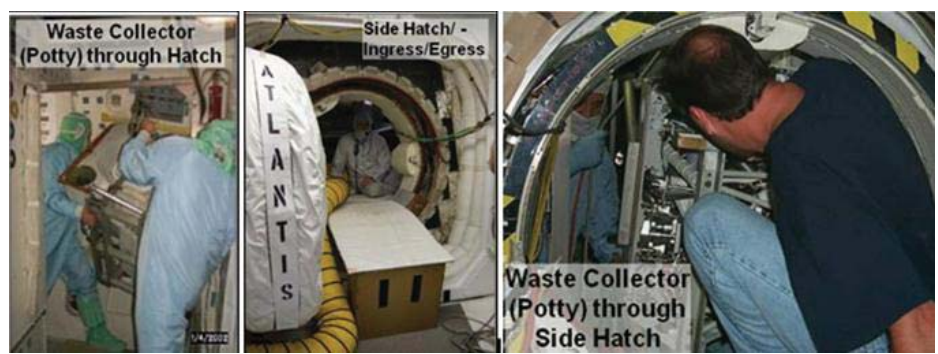

Figure 2 Hatch Ingress/Egress for Personnel/Hardware

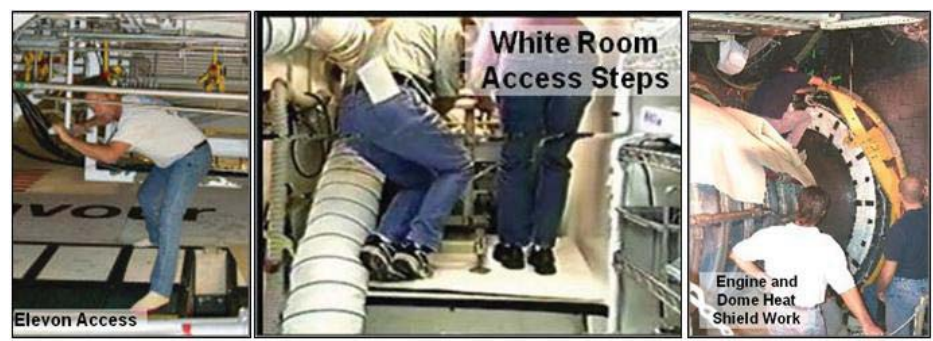

Figure 3 Uneven Surfaces

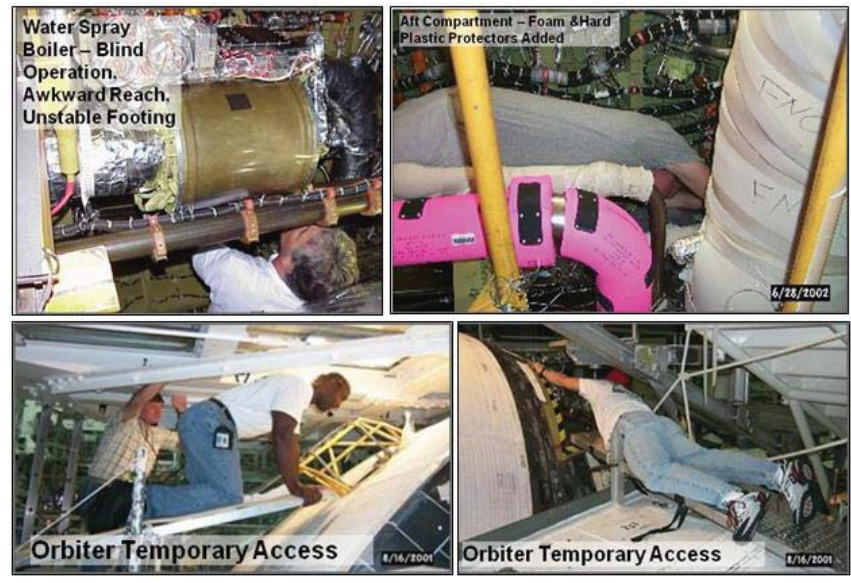

Figure 4 Prone Reach

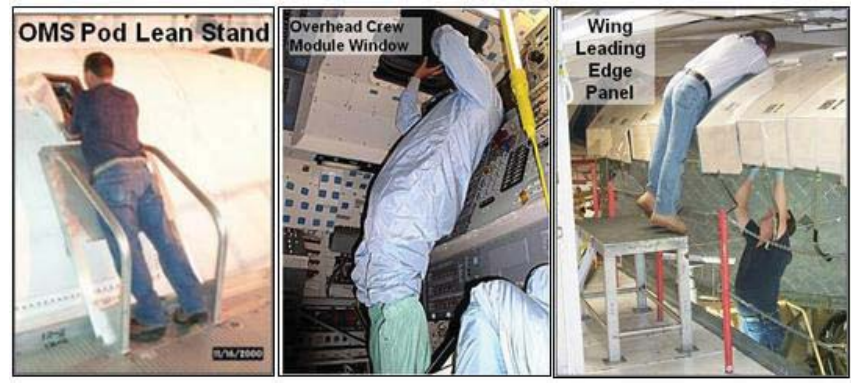

Figure 5 Leaning / Overhead Reach

\section{GROUND SYSTEMS DEVELOPMENT AND OPERATIONS}

GSDO operations affect various KSC facilities and landing and recovery operations in different states. GSDO provided 
information and candidate items with safety of ground crew being significant. During the upcoming years, the low frequency launch rate is predicted to further impact need for emphasizing human factors inputs and $\mathrm{M} \& \mathrm{~S}$ support.

The main Integrated Program Team (IPT) and Engineering Integration Team (EIT) of GSDO were reviewed for applicability to project efforts and human factors needs:

- Vehicle Integration and Launch Capabilities - Small Class Vehicle; Crawler Transporter; Mobile Launcher; Vehicle Assembly Building; and Launch Pad

- Command Control Communication and Range - End-to-End Command and Control; Communications Systems; Advanced Ground systems Maintenance; and Range Systems

- Offline Processing and Infrastructure Capabilities Environment and Infrastructure; Orion Production Operations; Spacecraft Offline Processing; Landing and Recovery; and Launch Vehicle Offline Processing

Design visualizations accompany the GSDO efforts. Through Ground Operations Planning Databases and documents, overall tasks will be shown and broken down to educate reviewers and those affected by GSDO. Humans may be placed in the M\&S environments, primarily showing vicinity and size aspects as well as basic motions near and around facility platforms and hardware. Detailed human factors analyses, such as hand grasp and ergonomic risks, will be included in future projects. $M \& S$ Designers for the GOPD and GSDO were identified as end-users for the Primitives Library.

\section{REQUIREMENTS}

Requirements within the following were reviewed for applicability to postures and motions that could be collected. Items within NASA-STD-3001 Volume 2, the Human Factors Engineering Analysis Tool based on FAA standards, and the Human Systems Integration Requirement (HSIR) were evaluated and included within the task selection and primitives identification. Both the NASA-STD-3001 Volume 2 and the HSIR are significant to the GSDO, MPCV, and SLS Programs.

\section{ORION TIMELINE ANALYSIS}

A timeline of events associated with several KSC areas and stages of the Orion spacecraft, prior to launch, was developed, so that each activity could be analyzed by operability and human factors experts with spacecraft processing experience. The Orion processing timeline included delivery from the Orion assembly line (located at KSC), Orion processing in Multi Purpose Processing Facility (MPPF), Orion/Ares rocket integration and testing in the Vehicle Assembly Building (VAB), Pad operations, Landing and Recovery as well as Post Flight processing.

The results of the human factors operability Timeline Analysis for Orion including processing improvement opportunities were reviewed by the team for potential impacts to task selection for the HF Primitives Library.

\section{Ground Operations Planning Database (GOPD)}

The GOPD consists of task information compiled in a web-based user interface database used to capture ground operations planning information. Content is developed by IPTs/EITs comprised of GSDO support in partnership with MPCV and SLS Program Support. It was developed on a capability-driven framework with multiple customers/users in mind. For some entities supporting, formal requirements exist. Others may be based on commercial activities with only NASA oversight. Thus, the goal is to build generically when possible to accommodate various options for spacecraft vehicles within the integration, rotation, and launch facilities. Focus on operability, including successes and changes to vehicle to help ground operations, has been a key factor, which was noted as also having an affordability factor including time and lower costs.

From discussions with the ground project, design and systems engineers, several areas of concern were established and listed for inclusion into the Primitive database. The following Figures 6 to 8 are examples of GOPD concerns.

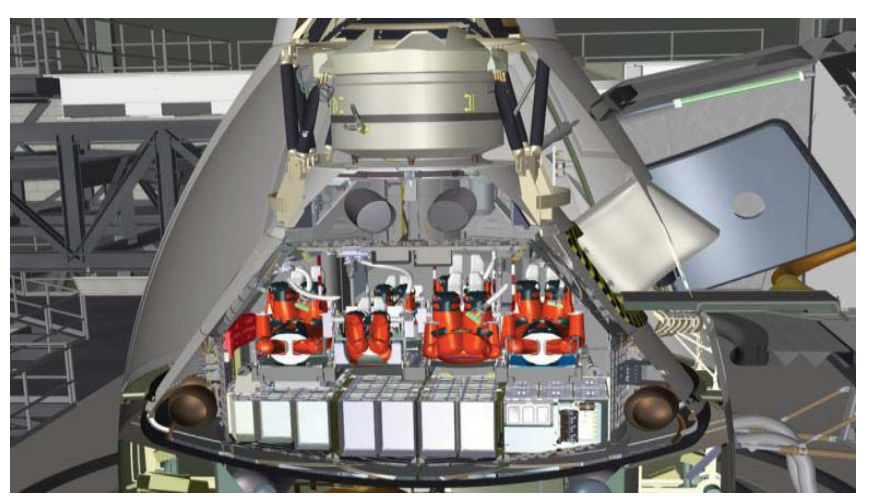

Figure 6 Crew Access Arm Ingress/Egress

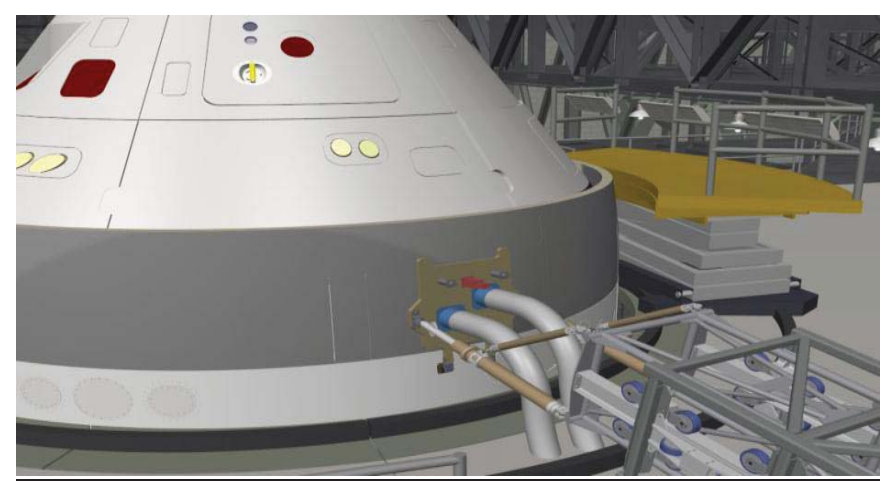

Figure 7 Installing/Removing the GSE side umbilicals 


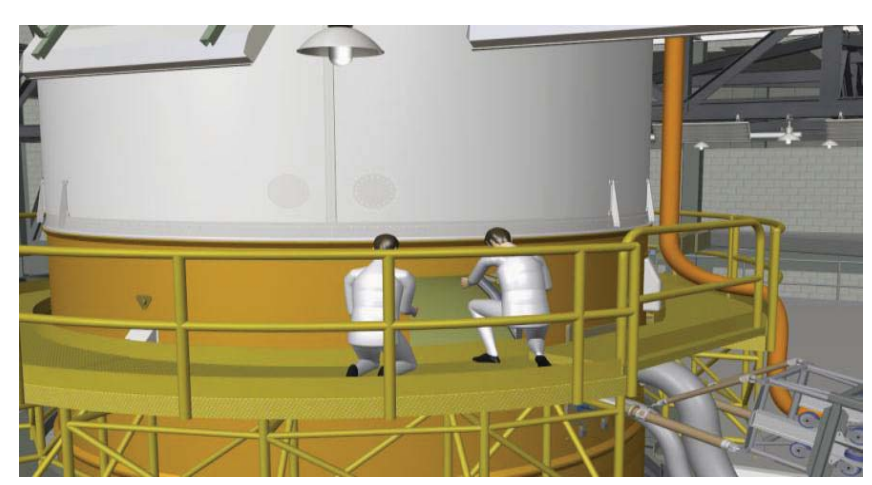

Figure 8 SLS Access Locations

\section{GSE DESIGN - ORION/MPCV ASSEMBLY}

Prior to the Orion Timeline activities at KSC, the Orion/MPCV will be assembled in the Operations and Checkout Building at KSC. A list of Orion Crew and Service Module Assembly candidate tasks were provided by GSE Design for consideration. A total of eleven tasks were identified, including several that were identified as potential areas of concern for Orion processing.

\section{NeXT STEPS}

Currently the project has established the plan to go forward with this project, as well as a comprehensive list of tasks to be performed. Pilot studies of motions have been completed, and the results of these studies have been included into the library. Statistical analysis is being performed for that pilot test of primitive motions. Thus the next steps are to begin performing the motion primitive captures, and populate the library.

\section{SUMMARY}

For many years the motion capture capabilities have been improving at KSC and MSFC along with the human systems engineering abilities. Now it is being recognized, after many years, that spacecraft processing includes many specialized activities requiring unique postures when handling delicate expensive hardware with high consequence of loss of mission if not processed correctly. Thus, the two are set in place, and it is critical to ensure that this ground processing knowledge is captured in the human motion primitive library for use by future designers of space launch systems.

\section{REFERENCES}

[1] Eric J. Schafer, Gena Henderson and Damon Stambolian. System Engineering Processes at Kennedy Space Center for Development of SLS and Orion Launch Systems. 2013 IEEEACpaper\#2686

[2] Katrine Stelges, Brad A. Lawrence. Human Engineering Modeling and Performance: Capturing Humans and Opportunities. Copyright (C) 2012 by United Space Alliance, LLC Kennedy Space Center, FL; USA
[3] Damon B. Stambolian, Brad A. Lawrence, Katrine S. Stelges, Marie-Jeanne O. Steady Ndiaye USA KSC, Ridgwell, Robert E. Mills, Gena Henderson, Donald Tran and Tim Barth, NASA Engineering and Safety Center (NESC). Human Modeling for Ground Processing Human Factors Engineering Analysis. IEEEACpaper\#1075

[4] Michael Bell, Gena Henderson and Damon Stambolian Lessons Learned for Improving Spacecraft Ground Operations. 2013 IEEEACpaper\#2698

\section{ACKNOWLEDGEMENTS}

The project success was significantly impacted by the support of the NASA Engineering and Safety Center (NESC), particularly Dr. Cynthia Null, the NESC Human Factors Technical Fellow, as well as key NESC stakeholders and team members at various NASA centers. The center-to-center communications including support of the motion capture facilities and skills at KSC and MSFC created collaborative expertise for effective planning, execution, and user deliverables. We would also like to acknowledge these other key contributors to the project: Lora C. Ridgwell, Robert E. Mills, Donald Tran, and Tim Barth, at KSC; along with Vicky Garcia, Caitlyn Durham, Jerry Wells, Mark Blasingame, Jason Quick, Jack Stokes, and Stephen Patrick at MSFC; and Dr. Barbara Kanki, Ben VanLear, and Dr. Fayssal Safie, members of the project's Steering Committee. 
